\title{
A numerical study of the 2:1 planetary resonance
}

\author{
F. Marzari ${ }^{1}$, H. Scholl ${ }^{2}$, and P. Tricarico ${ }^{3}$ \\ 1 Dipartimento di Fisica, University of Padova, via Marzolo 8, 35131 Padova, Italy \\ e-mail: marzari@pd.infn.it \\ 2 Observatoire de la Côte d'Azur, BP 4229, 06304 Nice Cedex 4, France \\ e-mail: scholl@obs-nice.fr \\ 3 Department of Physics, Washington State University, PO Box 642814, Pullman, WA, 99164-2814, USA \\ e-mail: tricaric@wsu.edu
}

Received 7 December 2005 / Accepted 9 February 2006

\section{ABSTRACT}

\begin{abstract}
We numerically explore the long-term stability of planetary orbits locked in a 2:1 mean motion resonance for a wide range of planetary mass ratios and orbital parameters. Our major tool is Laskar's frequency map analysis. Regions of low diffusion rate are outlined in a phase space defined by the two planetary eccentricities and the libration amplitude of a critical resonance argument. Resonant systems that are dynamically stable on a long timescale must lie within these regions. The resonance locking between planets in high eccentric orbits may be destroyed by mutual close encounters. We discuss various dynamical protection mechanisms related to the resonant configuration, among which is the well-known apsidal corotation. In the case of moderate-to-low eccentricities, we find that apsidal circulators, little discussed till now, are very common among stable orbits. We also map the different types of resonant behaviour predicted by analytical theories in the phase space.
\end{abstract}

Key words. celestial mechanics - planets and satellites: general - methods: $N$-Body simulations

\section{Introduction}

During the process of planetary formation, different dynamical mechanisms can lead planets to cross low-order meanmotion resonances and, eventually, to be subsequently captured into resonance. Nebula induced differential migration (Ward 1997; Goldreich \& Tremaine 1980; Lin \& Papaloizou 1986; Tanaka et al. 2002; Kley 2003), gravitational scattering of planets in crossing orbits (Rasio \& Ford 1996; Weidenschilling \& Marzari 1996; Moorhead \& Adams 2005), or migration caused by exchange of angular momentum with a debris disk of small planetesimals (Murray et al. 1998; Tsiganis et al. 2005), can all move planets from their original formation site into new orbits.

Planetary orbits may diverge or converge during migration. In both cases, planetary eccentricities may be enhanced. For diverging orbits, Chiang et al. (2002) propose repeated resonance crossings to reach the presently observed large planetary eccentricities. It may happen that two planets on converging orbits are captured into a mean motion resonance (e.g. Murray \& Dermott 1999). While the two planets continue to migrate, their eccentricities can be pumped up (e.g. Lee \& Peale 2002; Beaugé et al. 2003; Kley et al. 2005; Moorhead \& Adams 2005), eventually resulting in a destabilization of their orbits after a close encounter (Moorhead \& Adams 2005). Planets may remain on long-term stable orbits in resonance if the migration is halted before eccentricities that are too high are reached or if particular protection mechanisms related to the resonance locking avoid close encounters even when large eccentricities are reached. Depending on the migration speed and on the way in which it is modelled in the numerical simulations, the eccentricities may also reach a plateau while the planets are in resonance. In this case, even if the orbital decay continues, the planets never reach an unstable configuration (Lee \& Peale 2002; Nelson \& Papaloizou 2002; Beaugé et al. 2006).

Exoplanetary systems with resonant planets are of particular interest since they are good candidates for testing various migration scenarios. For this purpose, it is important to determine the regions of long-term stability for a wide range of parameters. Three resonant planetary systems orbiting about main sequence stars now appear to be well-established. The stars GJ 876 and HD 82943 have planets locked in a 2/1 resonance, while two of the four planets orbiting $55 \mathrm{Cnc}$ are in a $3 / 1$ resonance. In this paper, we concentrate on $2 / 1$ resonant systems.

A considerable amount of analytical work has been done on the secular dynamics of two planets in orbital resonance. Using the Hamiltonian formulation for the secular dynamics of the general three-body problem (e.g. Laskar \& Robutel 1995), Beaugé \& Michtchenko (2003) derive a canonical system for the planar resonant problem with two degrees of freedom by averaging over the rapidly changing angular variables. The system has stationary solutions, which means that intrinsic variables of the system are constant with time. For each planet, a combination of its semimajor axis and eccentricity is such an intrinsic variable, as does the difference between the periastron longitudes of the two planets. The stationary solutions are an excellent basis for exploring in a phase space defined by planetary eccentricities, mass ratios, and periastron longitudes, the dynamical behaviour of orbits in resonance in the frame of the averaged planar problem (Beaugé et al. 2003; Beaugé \& Michtchenko 2003; Ferraz-Mello et al. 2005b). The stationary solutions can be considered to form a skeleton for the set of all orbits in the planar non-averaged problem, also possibly for small inclinations. One may predict the behaviour of a large set of orbits in the nonaveraged problem over a certain interval of time from the behaviour of the stationary solutions. However, their stability on 
long time scales is not known even if stationary solutions are, of course, good candidates for long lasting resonant systems.

The stationary solutions of the averaged problem are characterized by apsidal corotators (ACRs). The difference between the direction of the apsidal lines of two planets remains constant. Since the difference between the periastron longitudes of two resonant planets is an intrinsic variable of the problem, the apsidal corotators are automatically found. In the non-averaged case and also for small inclinations, one can expect apsidal corotators to oscillate with small amplitudes around a fixed value. Apsidal corotators do not exclusively occur at resonances. On the contrary, as Michtchenko \& Malhotra (2004), have shown, they are very common outside of resonances in particular at high planetary eccentricities. At lower eccentricities, apsidal circulators dominate.

The success of the resonant stationary solutions in finding apsidal corotators may lead to the wrong assumption that nonapsidal corotators (NACRs, for Non-Apsidal CoRotation) are unstable and that apsidal corotation is always a necessary condition for long-term (Gyr) stability. As we show below, both assumptions are only true for very large eccentricities of both planets. For small to moderate eccentricities, there are both ACR and NACR solutions that are stable on long timescales implying that real planetary systems can be found in either of these states. The reason why the analytical work is usually restricted to ACR is related to the inherent difficulties in constructing analytical theories describing the NACRs behaviour, and not to a lack of knowledge of their existence.

Since, as outlined above, the stationary solutions of the averaged problem are an excellent basis for estimating the behaviour of orbits in the full problem over a certain period of time but not necessarily over timescales of Gyr, we apply a numerical method to investigating the long-term stability of planetary orbits in the 2/1 resonance. For a wide range of planetary mass ratios and orbital parameters, we apply Laskar's Frequency Map Analysis (FMA) to numerically integrated orbits. The starting planetary orbital elements are chosen at random and a total of $1.5 \times 10^{5}$ resonant planetary systems are integrated over $1 \mathrm{Myr}$.

We use the FMA method since it is a very sensitive tool for measuring the diffusion rate for each resonant system, a strong indicator of its long-term stability. The diffusion coefficients are presented in a phase space defined by two "free" planetary eccentricities and by the libration amplitude of a critical resonance argument. The corresponding diagrams are called diffusion maps showing the regions where stable resonant systems can be found. Our approach is substantially different from that of Godzdziewski et al. (2002), Bois et al. (2003), and Ji et al. (2004). Godzdziewski et al. (2002) and Bois et al. (2003) use MEGNO, a recent numerical tool for the detection of chaos, which was invented by Cincotta \& Simó (2000) to investigate the stability of planetary orbits in the proximity of the best-fit orbital solutions of the observed systems GJ 876 (Gozdziewski et al. 2002) and HD 160691 (Bois et al. 2003). Ji et al. (2004) perform numerical integrations of the planetary system HD 82943 for $10 \mathrm{Myr}$ to evaluate the stability of orbits near the nominal values of the orbital parameters. We instead perform a global analysis of the $2: 1$ commensurability by fully sampling the phase space at the resonance and by exploring the dependence of the results on the mass ratio between the central star and the planets. We also exploit the FMA method to derive orbital parameters other than the osculating ones that are more suited for a dynamical exploration of the phase space.
The FMA algorithm is outlined in Sect. 2 where the diffusion maps for different planetary mass ratios are also shown. Section 3 is devoted to a discussion of different protection mechanisms related to the resonance locking that prevent close planetary encounters for high eccentric orbits. In Sect. 4 we discuss the different types of resonant behaviour, giving particular attention to the case of NACRs that are as stable and frequent as ACRs. In the same section we compare our results for ACRs with the stationary solutions found by Ferraz-Mello et al. 2005a. In addition, we investigate the so-called asymmetric ACRs in more detail, which reveal concentrations around $60^{\circ}$ and $300^{\circ}$ for the critical resonance argument, respectively.

All our results are derived within the full planar three-body problem. The extension of these results to the inclined case will be the subject of a forthcoming paper.

\section{Regions of stability at the $2 / 1$ resonance}

\subsection{The numerical algorithms}

The FMA method (Laskar et al. 1992; Laskar 1993a; Laskar 1993b; Sidlichovský \& Nesvorný 1997), yields a quantitative measure of the stability of an orbit by determining the diffusion rate of intrinsic frequencies in Fourier space. An orbit is integrated numerically and the resulting orbital elements are Fourier analyzed. Values of intrinsic frequencies are obtained over running windows and their relative changes are estimated. A comparatively short integration period is necessary to measure the long-term stability of an orbit. The methodology used to perform the FMA on time series of the orbital elements of two planets in resonance is described in detail in Marzari et al. (2005). When applying the FMA method to a given problem, the choice of the variables for the Fourier analysis and for determining of the diffusion rate is not necessarily straightforward and may require some particular attention. Otherwise, the chosen signal might be artificially blurred by a bad choice of variables eventually masking a slow diffusion or, on the contrary, suggesting a false diffusion.

Using SYMBA (Duncan et al. 1998), we numerically integrate a large number of planetary systems $\left(\sim 1.5 \times 10^{5}\right)$ in $2: 1$ mean motion resonance. The mass of the central star is assumed to be $1 M_{\odot}$ in all our simulations and the two planets are Jovian type planets. One planet always has a mass equal to 1 Jupiter mass, while the mass of the other is varied in order to give different mass ratios $\mu=m_{2} / m_{1}$ where $m_{1}$ is the mass of the inner planet. Seven different values of $\mu$ are considered in our simulations, ranging from 0.1 to 10 . For larger (or smaller) mass ratios, the problem approaches the well-known restricted threebody problem. The semimajor axis of the inner planet is fixed and set equal to $5 \mathrm{AU}$. The semimajor axis of the outer planet is chosen at random within an interval located near $8 \mathrm{AU}$ and centered at the nominal resonance location for that particular value of $\mu$. The interval is always broader than the resonance width, but only systems that are in the 2:1 resonance for at least $1 \mathrm{Myr}$ are retained and analyzed with the FMA. Systems out of resonance or unstable on a short timescale are then discarded. The residence in resonance is verified from analysing the resonant arguments performed on-line during the numerical integration. Results for semimajor axes other than $5 \mathrm{AU}$ for the inner planet scale easily. All orbits have an inclination of $0^{\circ}$. Angular variables for the planets are chosen at random between $0^{\circ}$ and $360^{\circ}$. The starting eccentricities are randomly distributed between 0 . and 0.9. With this Montecarlo approach, we fill the phase space at the resonance densely. The integration period of $1 \mathrm{Myr}$ is 
sufficiently long to build the necessary time series of the orbital elements for the FMA method.

With the Fourier method of the FMA (1997), we analyze the signal:

$s_{\Delta \varpi}=\cos \left(\varpi_{1}-\varpi_{2}\right)+i \sin \left(\varpi_{1}-\varpi_{2}\right)$.

The diffusion rate $\sigma_{\text {FMA }}$ of the main Fourier component of $s_{\Delta \varpi}$ is measured as the negative logarithm of the standard deviation of $s_{\Delta \varpi}$ on running windows of $10^{5}$ years over the entire integration timespan of $1 \mathrm{Myr}$. Each running window includes enough periods of $\Delta \varpi$ for a meaningful Fourier analysis. The variable $\Delta \varpi$ either circulates or librates. We prefer to apply the FMA to the signal $s_{\Delta \varpi}$ rather than to the more conventional signals $s_{i}=h_{i}+i k_{i}, i=1,2$, where $h_{i}=e * \cos \left(\varpi_{i}\right)$ and $k_{i}=e * \sin \left(\varpi_{i}\right)$. When the system is in apsidal corotation, both $s_{1}$ and $s_{2}$ may have variations related to the corotation libration and not to chaotic diffusion.

Slow diffusion rates (large $\sigma_{\text {FMA }}$ ) mean stable orbits on longer periods of time than in the case of fast diffusion (small $\sigma_{\text {FMA }}$ ). The FMA gives a relative measure of stability. Additional simulations over Gyr are, therefore, necessary for a few selected values of $\sigma_{\text {FMA }}$ in order to know the stability period in years.

Diffusion maps are presented in a phase space defined by the "free" eccentricities $e_{1}$ and $e_{2}$ of the two planets and by a suitably defined libration amplitude of a critical resonance argument. The "free" eccentricities are obtained by a spectral decomposition of the two conventional signals $s_{1}$ and $s_{2}$. The $e_{1}, e_{2}$ can be considered as averaged eccentricities and can be compared with the eccentricities used in analytical, averaged models. For the $2 / 1$ resonance, there are two independent critical resonance arguments: $\theta_{1}=2 \lambda_{2}-\lambda_{1}-\varpi_{1}$, and $\theta_{2}=2 \lambda_{2}-\lambda_{1}-\varpi_{2}$, where $\lambda$ and $\varpi$ denote respectively mean longitude and longitude of periastron. While in resonance, at least one of the two resonance arguments librates. In the case of apsidal corotation both arguments librate as can be easily seen from the definition of $\theta_{1}$ and $\theta_{2}$. When only one argument librates (NACR), we use the librating $\theta$ to determine a libration amplitude of the resonance argument defined as the difference between the maximum and minimum values of $\theta$ averaged over running windows. In the case of ACR when both $\theta_{1}$ and $\theta_{2}$ librate, we define as the libration amplitude of the resonance that of the critical argument with lower libration amplitude. The critical resonance argument that is finally used for determining a libration amplitude is designated by $\theta_{\mathrm{m}}$. An average libration amplitude $D$ of $\theta_{\mathrm{m}}$ is computed as the mean of the maximum libration amplitude over sub-windows $\left(\Delta t=1 \times 10^{5} \mathrm{yr}\right)$ of the whole integration timespan. The choice of the sub-windows is made after a detailed analysis of the libration period of the two resonances in our simulations.

The FMA analysis yields for each orbit the variables $e_{1}, e_{2}$, $D, \sigma_{\mathrm{FMA}}$, the center of libration of $\theta_{\mathrm{m}}$, the corotation state, the amplitude and center of libration of $\Delta \varpi$ for ACR systems.

\subsection{Diffusion maps for different mass ratios}

As outlined above, the diffusion rate of a resonant system is quantified by the standard deviation $\sigma_{\mathrm{FMA}}$ of the signal $s_{\Delta \varpi}$. The diffusion rate can be presented in a three-dimensional phase space determined by the "free" planetary eccentricities $e_{1}, e_{2}$, and by the libration amplitude $D$. Since three-dimensional figures may hide important features due to a bad selection of the angle of view, we like to show diffusion maps projected on two of the three necessary variables. We show in Figs. 1 and 2 diffusion maps for five different values of $\mu$. These maps are projected in two significant planes: the plane of the two "free" eccentricities $\left[e_{1}, e_{2}\right]$, and that of $\left[e_{1}, D\left(\theta_{\mathrm{m}}\right)\right]$. The different grey coding in the maps indicates the local diffusion speed. Low diffusion speed (large values of $\sigma_{\mathrm{FMA}}$, light grey) implies regular behaviour and stability over a long timescale. High diffusion speeds (small values of $\sigma_{\mathrm{FMA}}$, dark grey) are typical of chaotic orbits that are stable only for a short timescale, inversely proportional to $\sigma_{\text {FMA }}$. Empty zones either do not allow stable orbits for at least $1 \mathrm{Myr}$, or they have a value of $\sigma_{\text {FMA }}$ lower than 0.5 , which means that the local planetary system is strongly chaotic.

In all five cases we notice a compact domain of stability for small eccentricities of the outer planet (planet 2) and a tail at high eccentricity. The tails roughly reproduce the isopleth structure for $\mu=$ const. as described in Ferraz-Mello et al. (2005a, see their Fig. 1.21). Their location in the eccentricity space strongly depends on $\mu$. Our numerical solutions show how these isopleths are more complex in structure as compared to the analytical predictions. As an example, for $\mu=0.1$ the stable solutions for low $e_{1}$ and large $e_{2}$ cover a wide area in the $\left[e_{1}, e_{2}\right]$ space. Moving towards higher values of $\mu$, the stable solutions at high values of $e_{2}$ split in different regions.

A major source of the instability of systems locked in resonance are close encounters between the two planets. Therefore, crossing orbits need particular attention. The dashed line in Fig. 1 marks the border line for crossing orbits, derived from the average eccentricity of the two planets. The number of systems above this line is significant and their stability can be maintained only by protection mechanisms related to the resonance. These mechanisms are discussed in the next section.

In Fig. 2 we show that stable resonant systems can be found even at large libration amplitudes up to $D \sim 300^{\circ}$, apart from the case with $\mu=0.1$. The regions with lower diffusion rate appear reasonably compact. The few scattered unstable cases are a projection effect of the 2D maps. In both Figs. 1 and 2 we mark with an empty circle the location of the extrasolar planetary systems GJ 876 and HD 82943. To compute $\left[e_{1}, e_{2}, D\right]$ and $\sigma_{\mathrm{FMA}}$ for GJ 876 we took the physical parameters and initial osculating orbital elements given in Laughlin \& Chambers (2001). The GJ 876 system appears to be embedded well in the stable region for $\mu=3.3$, and its $\sigma_{\mathrm{FMA}}$ is similar to that of surrounding systems on our map. For the resonant planets orbiting HD 82943, we use the orbital elements corresponding to solution A in Ferraz-Mello et al. (2005), who correct the originally published orbital elements that correspond to an unstable system. It is, therefore, not surprising that the resonant planetary system lies at the border of the stable area. It must be pointed out that the mass ratio between the star and the planets for both GJ 876 and HD 82943 is different from that of our 3-body problem where the mass of the central star is always $1 M_{\odot}$ and the mass of one of the two planets is $1 M_{\text {Jupiter }}$. The stability regions for systems with a mass ratio between the planets and the star equal to that of GJ 876 or HD 82943 might be smaller when compared to those found within our model. However, the values of $\sigma_{\mathrm{FMA}}$ for both the observed systems are high (this means stability) and similar to those of systems in our maps with close values of $e_{1}, e_{2}$, and $D$. This suggests that if there is any shrinking, this is not relevant enough to drive both systems out of the stable zone.

\section{Protection mechanisms while in resonance}

As shown in the previous section, a significant fraction of planetary systems that are stable according to the FMA analysis are 


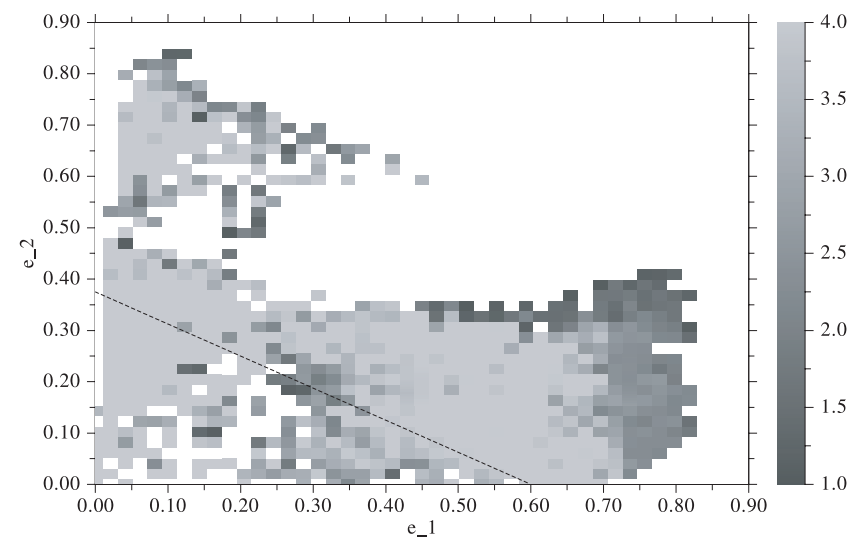

(a)

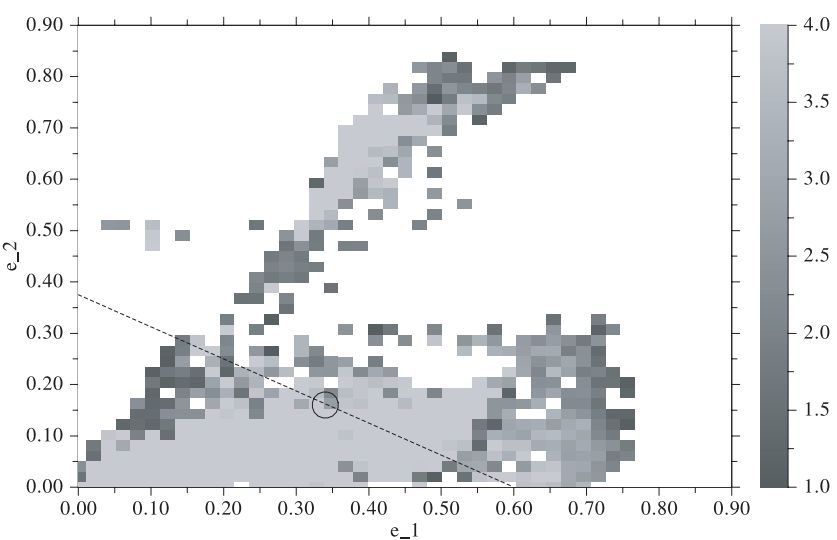

(c)

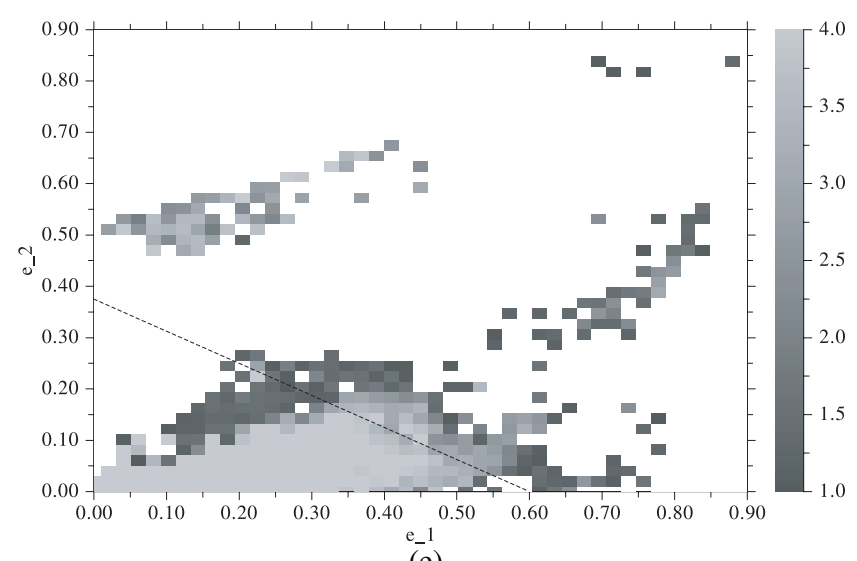

(e)

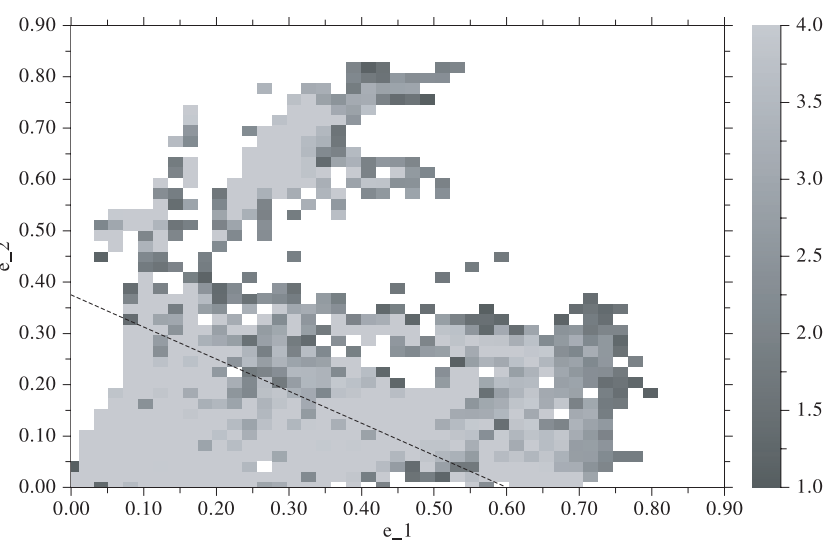

(b)

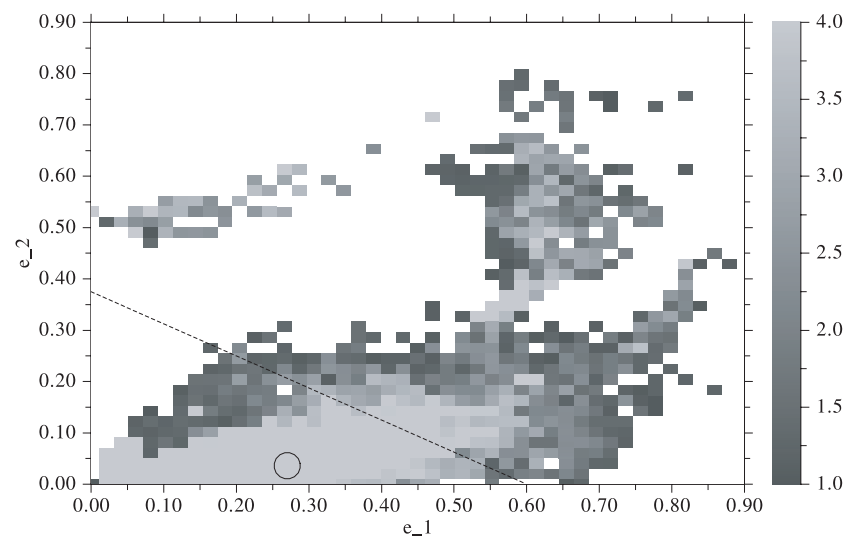

(d)

Fig. 1. Diffusion maps in the $\left(e_{1}, e_{2}\right)$ plane for different planetary mass ratios $\mu$. a) is for $\left.\mu=0.1, \mathbf{b}\right)$ for $\mu=0.6$, c) for $\mu=1.0$, d) for $\mu=3.3$, and e) for $\mu=10.0$. The grey levels correspond to different values of the diffusion speed measured by $\sigma_{\mathrm{FMA}}$. Regions with large values of $\sigma_{\mathrm{FMA}}$ (light grey) are stable over a long timescale. The dashed line in each plot marks the boundary between crossing and non-crossing orbits. The empty circle in the plot for $\mu=1.0$ shows the location in the map of the HD 82943 system, while that in the plot for $\mu=3.3$ represents the GJ 876 system.

in, or very close to crossing orbits. In our sample we identify four major modes of protection against close encounters allowing these systems to remain stable:

- Mode I: systems with anti-aligned periastra. In this configuration, the $2: 1$ ratio between the orbital periods easily protects the planets against mutual collisions. Our simulations show that the minimum distance between the two planets is reached when the inner planet is at periastron, while the outer planet is about half-way between periastron and apastron.

- Mode II: systems with aligned periastra. For small libration amplitudes of $\Delta \varpi$, the orbits may not intersect in spite of large values of $e_{1}$ and $e_{2}$.
By inspecting our sample of systems we also find two "weak" protection mechanisms:

- Mode III: since the eccentricities of the two planets oscillate in anti-phase, the eccentricity of planet 1 is at its maximum when that of planet 2 is at its minimum. This prevents close encounters in those systems where the mean eccentricities $e_{1}$ and $e_{2}$ of the two planets are just beyond the limiting value for crossing orbits.

- Mode IV: NACR systems can be on stable orbits with high eccentricities in absence of any periastron alignment. We find that in these cases the libration period of the critical argument is close to the circulation period of $\Delta \varpi$. 


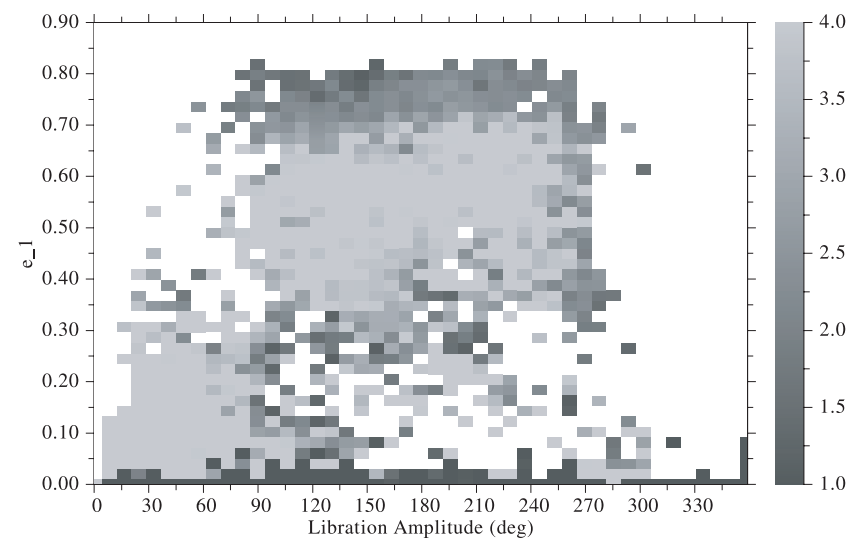

(a)

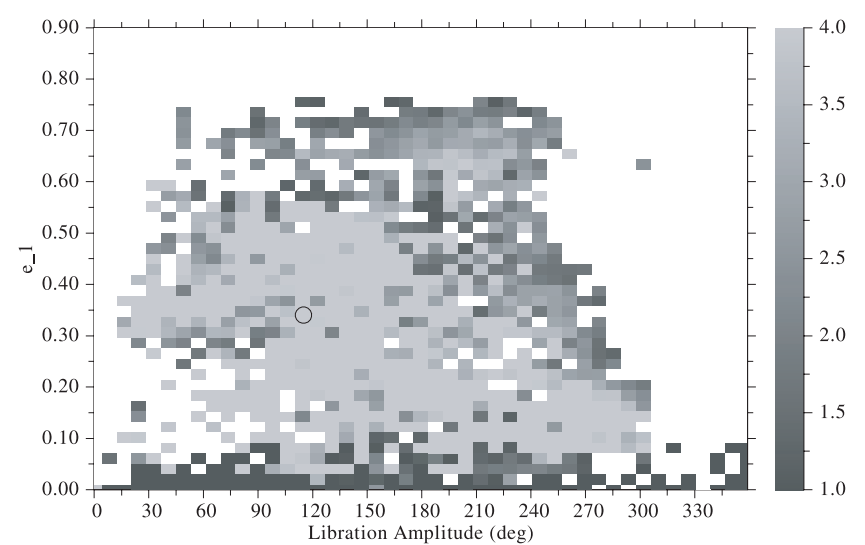

(c)

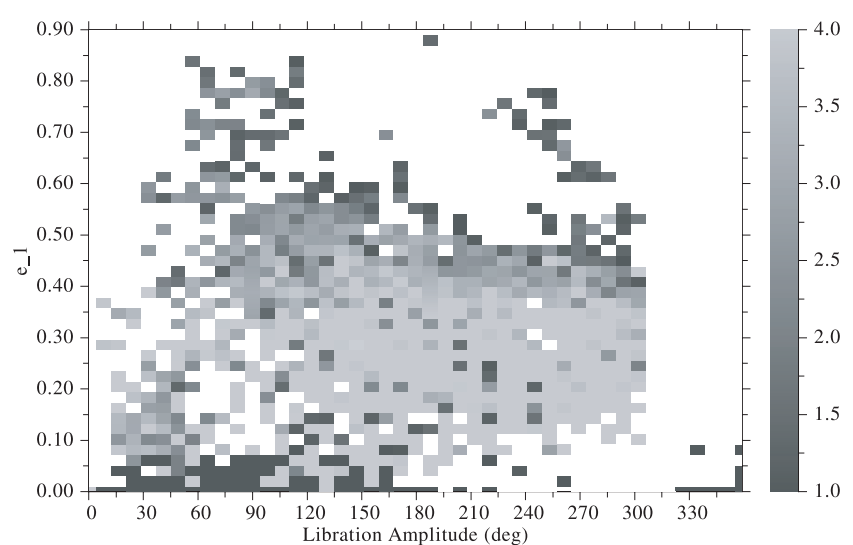

(e)

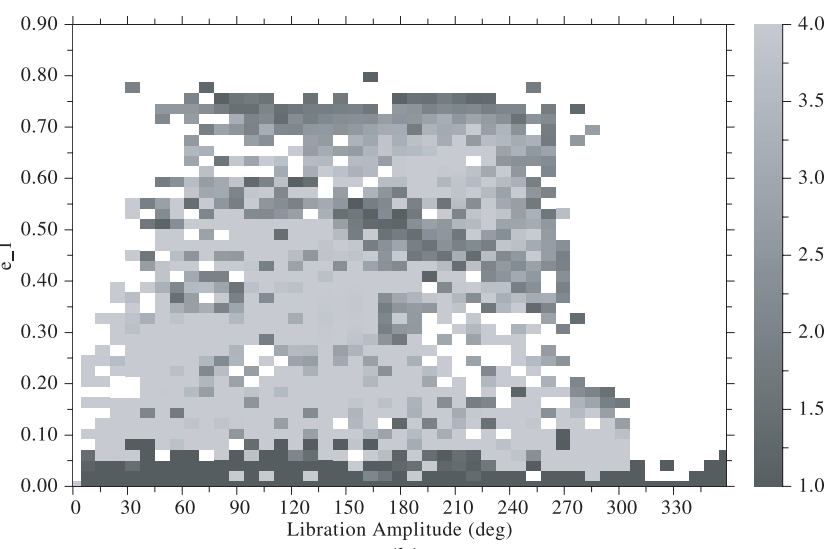

(b)

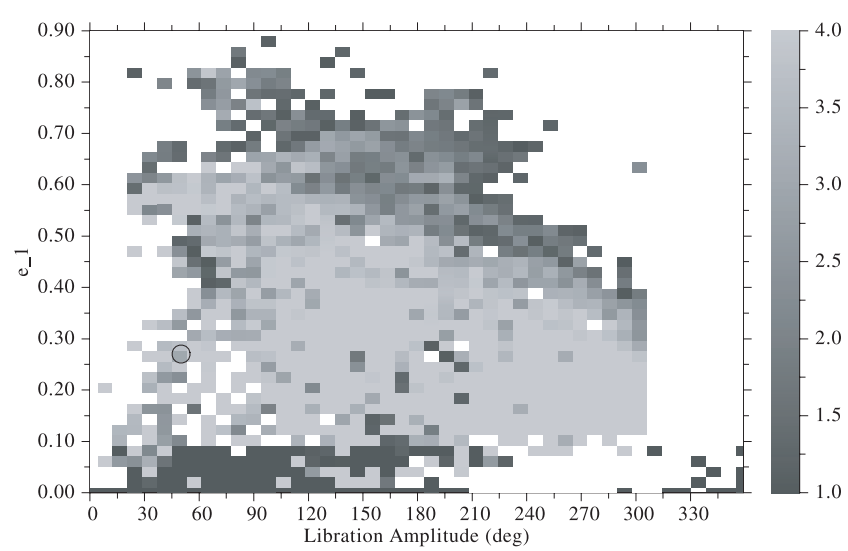

(d)

Fig. 2. Diffusion maps for the resonance in the $\left(e_{1}, D\right]$ plane for different planetary mass ratios $\mu$ as in Fig. 1. The empty circles show the location of the HD 82943 and GJ 876 systems.

This circulation period is correlated to the frequencies of eccentricity oscillations. As a consequence, the distance between the two planets can be increased by this particular frequency locking as compared to unlocked cases. An example of this behaviour is shown in Fig. 3; in spite of high eccentricities, and then deeply crossing orbits, the planets never get closer than 2.5 AU. We have verified that all these systems are not ACR systems in paradoxical circulation. (Ferraz-Mello et al. 2005c.)

We call these last two protection modes weak because, on average, the planets can get closer as compared to the cases of "strong" protection for similar orbital parameters.

\section{Locations of apsidal corotators in phase space}

The large number of numerical integrations we have performed allows us to map the location of different types of resonant behaviour in the phase space. Our results can be compared with the theoretical predictions of Ferraz-Mello et al. (2005a) to test their range of validity, which can be limited for the approximations in the analytical developments. Moreover, we can also explore new dynamical effects like those NACR cases not covered by the stationary solutions of the averaged method. We first compare the domain of systems in apsidal corotation with that of systems where the apsidal angle $\Delta \varpi$ circulates. We recall here that in these NACR systems only one critical angle librates, either $\theta_{1}$ or 

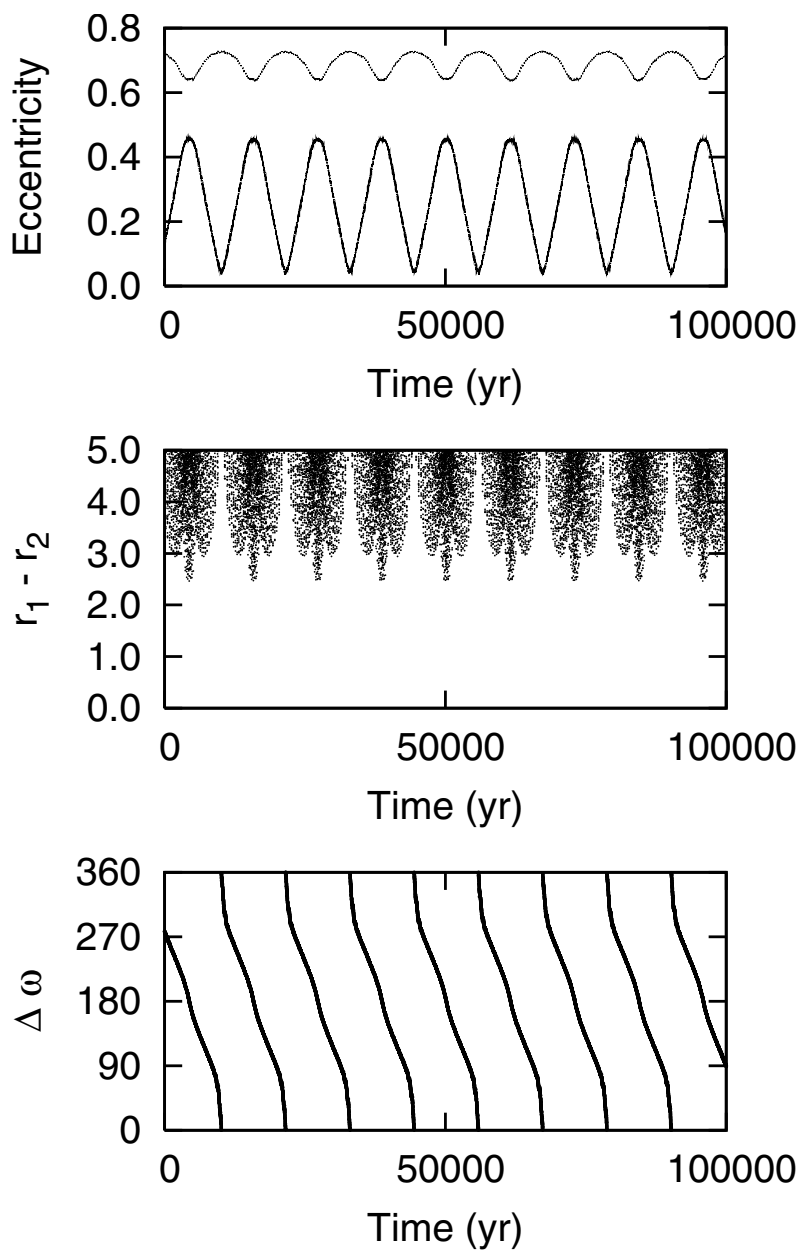

Fig. 3. Time evolution of eccentricities (dotted line is for the inner planet), mutual distance, and apsidal angle of two planets in resonance protected against close encounters by the Mode IV mechanism.

$\theta_{2}$. These domains appear to overlap in two-dimensional plots in the planes $\left(e_{1}, e_{2}\right),\left(e_{1}, D\right)$, or $\left(e_{2}, D\right)$ and no clear separation can be outlined by inspecting these figures. A tri-dimensional plot (Fig. 4) instead reveals a clear separation between ACR and NACR systems. NACR systems always have a higher value of $D$ than ACR systems for the same $e_{1}$ and $e_{2}$ values. In this plot we include resonant systems with all seven different values of the mass ratio $\mu(0.1,0.3,0.6,1.0,3.3,6.6,10.0)$.

The stability properties of ACRs and NACRs are not significantly different according to the FMA. A large fraction of NACR systems have very low diffusion rates suggesting long term stability of the order of some Gyr. Real planetary systems in a 2:1 resonance possibly detected in the future may, with equal probability, be either in an ACR or NACR state. However, the location in the phase space for ACRs and NACRs is different, and ACR states dominate at large eccentricities for both planets and low libration amplitude of the critical argument as shown in Fig. 4. This is the domain of crossing orbits and the stability of ACR resonant systems is reinforced by strong protection mechanisms described in the previous section (Mode I).

We can also compare the theoretical predictions of the Ferraz-Mello et al. (2005a), model (Fig. 1.20) with the outcomes of our integrations. We consider four ACR states in analogy with the definition of families of stationary solutions in Ferraz-Mello et al. (2005a). In the first case, both the apsidal angle $\Delta \varpi$ and the critical variable $\theta_{\mathrm{m}}$ librate around 0 : this state is designated

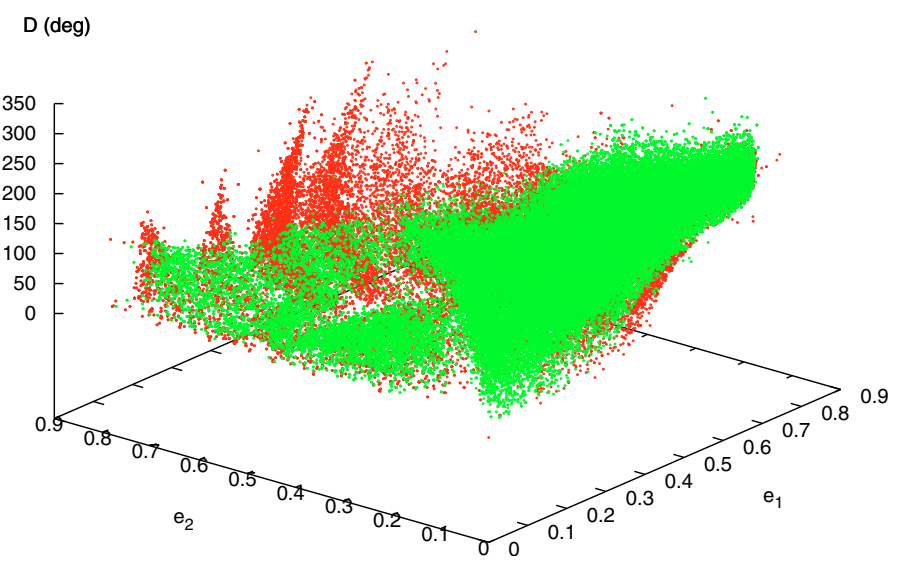

Fig. 4. Three-dimensional distribution of ACRs (red dots) and NACRs (green dots) in the $\left(e_{1}, e_{2}, D\right)$ space. The regions of different apsidal behaviour are clearly separated.

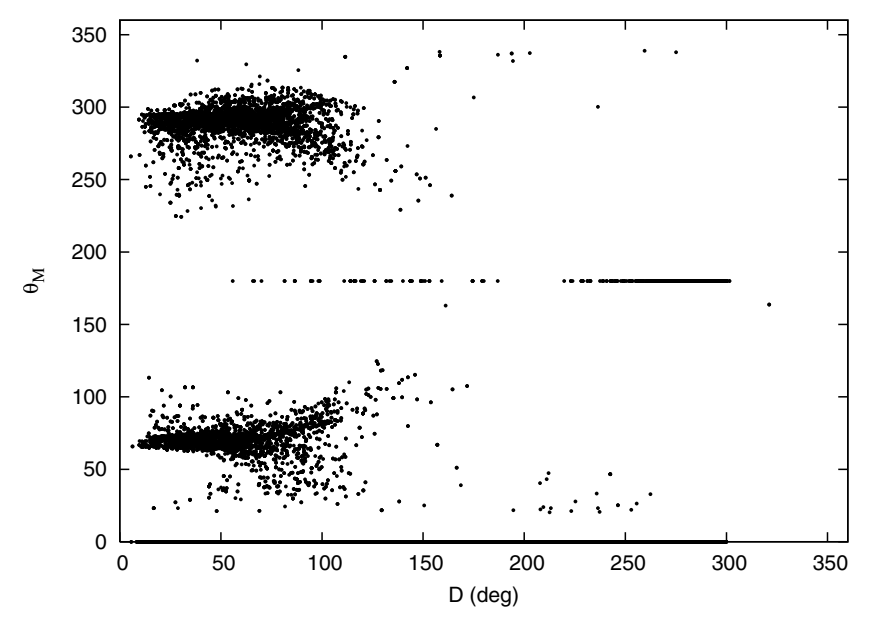

Fig. 5. Location of the asymmetric solutions in a plane defined by libration amplitude $D$ (in abscissa) and the libration center of the critical argument $\theta_{\mathrm{m}}$ : two swarms are clearly concentrating around $\theta_{\mathrm{M}} \sim 60^{\circ}$ and $\sim 300^{\circ}$ presumably forming a pitchfork at $D=180^{\circ}$; points concentrate also around $\theta_{\mathrm{M}}=0^{\circ}$ for all $D$ below $\sim 300^{\circ}$.

by $(0,0)$ following the notation of Ferraz-Mello et al. (2005a). In the second state, $(\pi, \pi)$, both $\Delta \varpi$ and $\theta_{\mathrm{m}}$ librate around $\pi$. The state $(0, \pi)$ has $\theta_{\mathrm{m}}$ librating around 0 and $\Delta \varpi$ around $\pi$. Finally, we consider the antisymmetric cases, which we like to designate by (asy) hereinafter, where the center of libration is shifted away from either 0 or $\pi$. In Fig. 5 we show the location of the libration center as a function of the libration amplitude $D$ for all our systems. The (asy) states are concentrated around $\theta_{\mathrm{M}} \sim 60^{\circ}$ and $\sim 300^{\circ}$ presumably forming a pitchfork at $D=180^{\circ}$; points also concentrate around $\theta_{\mathrm{M}}=0^{\circ}$ for all $D$ below $\sim 300^{\circ}$.

In order to show the separation between the different types of solutions clearly we use a three-dimensional plot. In Fig. 6 we illustrate the location of the classes of motion in the space defined by $\left[e_{1}, e_{2}, D\left(\Theta_{\mathrm{m}}\right)\right]$. It is noteworthy that the libration amplitude plays again an important role in shaping the regions populated by different states. For the sake of comparison with Fig. 1.20 in Ferraz-Mello et al. (2005a), we project the data in the $\left(e_{1}, e_{2}\right)$ plane (Fig. 7). The isopleth structure predicted by Ferraz-Mello et al. (2005a), for different mass ratios $\mu$ is populated by $(0, \pi)$ and (asy) states, with the latter slightly shifted with respect to the $(0, \pi)$ ones. Most of these states are on crossing orbits protected from close encounters by the Mode I protection mechanism. For large mass ratios, the isopleths at large 


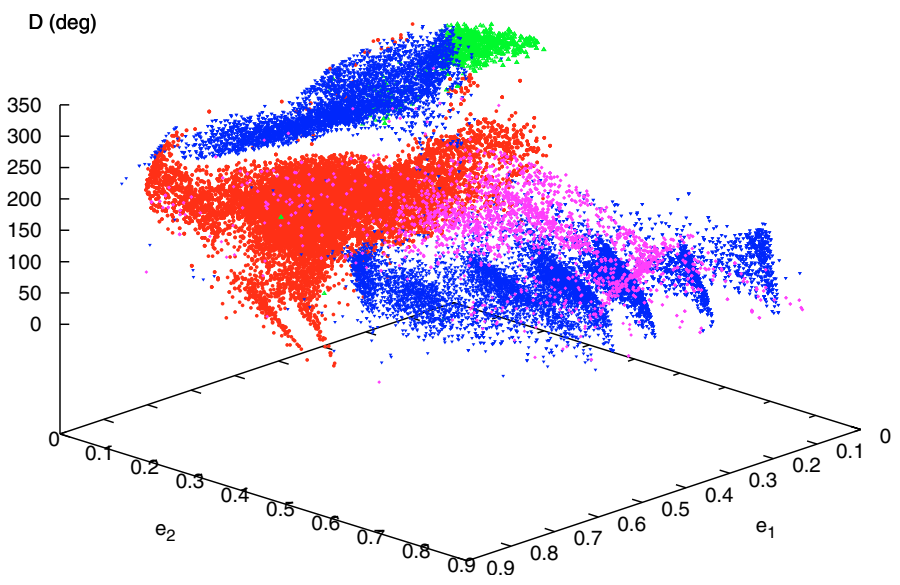

Fig. 6. Domains of the ACRs in the three-dimensional space $\left(e_{1}, e_{2}, D\right)$. The color coding is: 1$)$ red for $(0,0), 2)$ green for $(\pi, \pi), 3)$ blue for $(0, \pi)$ and 4$)$ magenta for asymmetric solutions.

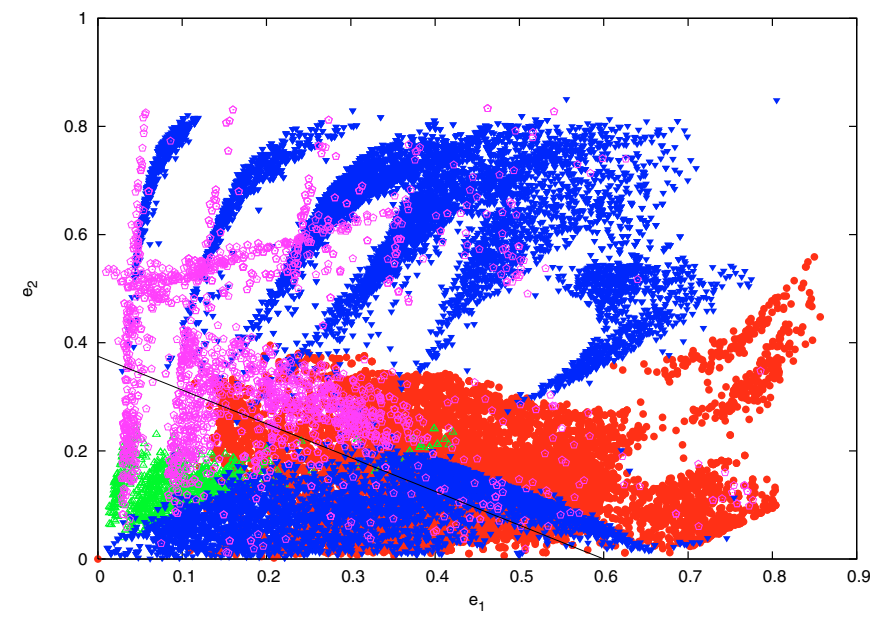

Fig. 7. Projection of the ACRs in the two-dimensional plane $\left(e_{1}, e_{2}\right)$. The color coding is the same as in Fig. 6 . The dark line marks the boundary between crossing and non-crossing orbits.

eccentricities are instead $(0,0)$ states where the Mode II protection grants stability. Systems close to the black line, the border between crossing and non-crossing obits, are protected by the weak mechanism related to the anti-phased oscillations of the planet eccentricities (Mode III). In this zone we find different types of states, $(0,0),(0, \pi),(\pi, \pi)$ and $($ asy).

Through the paper we use $\theta_{\mathrm{m}}$ as the critical argument of the resonance, while Ferraz-Mello et al. (2005a) always adopt $\theta_{1}$ in their formalism. To avoid confusion while comparing analytical results with numerical outcomes, we show the same plot in Figs. 8 as in 7 but, instead of using $\theta_{\mathrm{m}}$ to define the different ACR states, we switch to $\theta_{1}$ as they do. The main difference between the two plots appears at high eccentricity. In Fig. 8 the large eccentricity cases are now $(0, \pi)$ states while in Fig. 7 where $\theta_{\mathrm{m}}$ is used they are, as stated before, $(\pi, \pi)$ states. This means that for all these systems, $\theta_{2}$ is always the critical argument with a lower libration amplitude, and it librates around $0 . \theta_{1}$ instead librates around $\pi$ with a larger libration amplitude.

\section{Conclusions}

Our numerical analysis of the $2 / 1$ resonance shows that its structure is complex and that it strongly depends on three parameters: $e_{1}, e_{2}, D$. Apsidal corotation is a very important dynamical

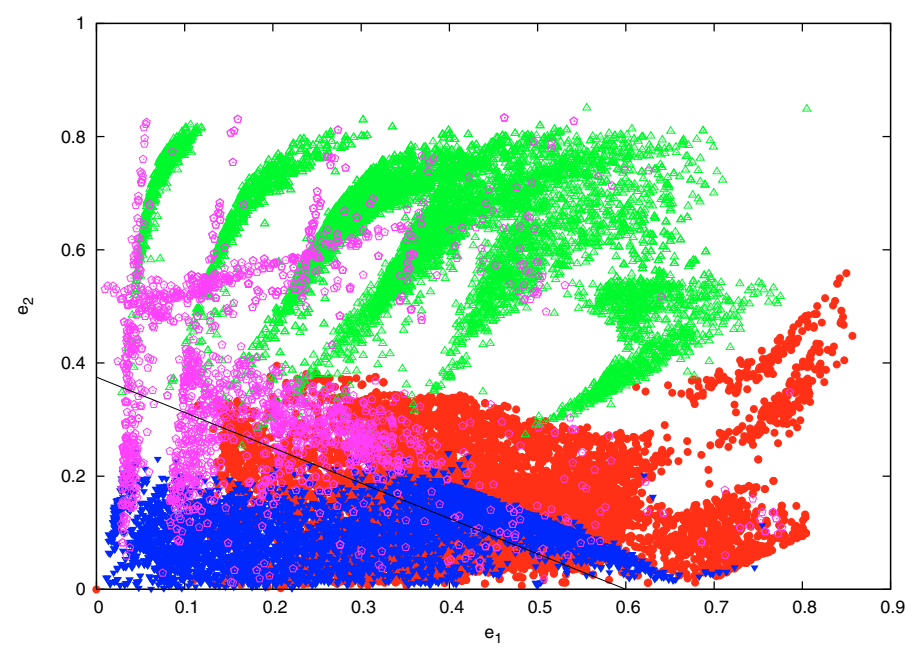

Fig. 8. Same as in Fig. 7 except that in the definition of the different resonant states instead of using $\theta_{\mathrm{m}}$ we adopt $\theta_{1}$ as in Ferraz-Mello et al. (2005a). At high eccentricity the $(0, \pi)$ states are now defined as $(\pi, \pi)$ states.

feature, but it is not an essential ingredient for the long-term stability of planetary orbits with low-to-medium values of eccentricities. Apsidal corotation implies the simultaneous libration of both critical resonance arguments $\theta_{1}$ and $\theta_{2}$. The NACR systems, characterized by the libration of a single critical argument, fill up the phase densely space and have similar diffusion rates to ACR systems. At high eccentricities the ACRs dominate since their stability against close encounters is granted by strong protection mechanisms related to apsidal corotation and resonance locking. Otherwise, the separation between these two classes of solutions is governed by the libration amplitude of the critical argument. Concerning the ACRs, we find very good agreement with the results of Ferraz-Mello et al. (2005a), based on the averaged theory of Beaugé et al. (2003). Discrepancies are of very minor importance and presumably caused by the restrictions within the theory. The asymmetric ACRs do not have a uniform distribution for their libration center but are concentrated symmetrically around $\sim 60^{\circ}$ and $\sim 300^{\circ}$.

Which types of systems in the $2 / 1$ resonance can be expected? From the purely dynamical point of view, systems with very wide ranges of planetary mass ratios and orbital parameters show long-term stability. ACRs and NACRs have an equal probability. For large eccentricities of both planets, ACRs dominate very clearly. The resonant systems that will be detected in the future may not fill all the phase space of long-term stable orbits. Planets are believed to form on almost circular orbits. Additional processes bring them on higher eccentric orbits, as outlined in the introduction. In particular, migration scenarios are presently proposed. When two planets on converging orbits are captured in a resonance due to migration, their eccentricities build up (e.g. Lee \& Peale 2002; Beaugé et al. 2003; Kley et al. 2005; Moorhead \& Adams 2005), and ACRs appear to be obtained right after capture. They may also evolve into NACRs like the $3 / 1$ resonant planets $b$ and $c$ orbiting Cnc55 (Marzari et al. 2005). More systematic investigations are necessary to explore the final configurations of resonant systems captured in resonance by migration in order to make more precise predictions. Concerning final eccentricities, there is agreement on the fact that the less massive planets usually gain higher eccentricities than the more massive ones (e.g. Moorhead \& Adams 2005). Comparing the dynamical 
characteristics of detected resonant systems in the future with explorations of regions of long-term stability and simulations of capturing planets in resonance will provide a basis for testing various scenarios for the origin of resonant systems.

Acknowledgements. We thank Cristian Beaugé for useful comments and suggestions while acting as referee.

\section{References}

Beaugé, C., \& Michtchenko, T. A. 2003, MNRAS, 341, 760

Beaugé, C., Ferraz-Mello,S., \& Michtchenko, T. A. 2003, ApJ, 593, 1124

Beaugé, C., Michtchenko, T. A., \& Ferraz-Mello, S. 2006, 365, 1160

Bois, E., Kiseleva-Eggleton, L., Rambaux, N., \& Pilat-Lohinger, E. 2003, ApJ, 598, 1312

Chiang, E. I., Fischer, D., \& Thommes, E. 2002, ApJ, 564, L105

Cincotta, P., \& Simoó, C. 2000, A\&AS, 147, 205

Duncan, M. J., Levison, H. F., \& Lee, M. H. 1998, AJ, 116, 2067

Ferraz-Mello, S., Michtchenko, T. A., Beaugé, C., \& Callegari, N. 2005a, in Chaos and stability in extrasolar planetary systems, ed. R. Dvorak, F. Freistetter, \& J. Kurths, preprint

Ferraz-Mello, S., Michtchenko, T. A., \& Beaugé, C. 2005b, in Chaotic worlds: from order to disorder in gravitational N-body system, ed. R. Dvorak, F. Freistetter, \& J. Kurths, preprint

Ferraz-Mello, S., Michtchenko, T. A., \& Beaugé, C. 2005c, ApJ, 621, 473
Gozdziewski, K., Bois, E., \& Macieijewski, A. J. 2001, MNRAS, 332, 839 Goldreich, P., \& Tremaine, S. 1980, ApJ, 241, 425

Ji, J., Kinoshita, H., Liu, L., Nakai, H., \& Li, G. 2004, submitted [arXiv:astroph-0403386]

Kley, W. 2003, Cel. Mech., 87, 85

Kley, W., Lee, M. H., Murray, N., \& Peale, S. J. 2005, A\&A, 437, 727

Laskar, J. 1993a, Physica D, 67, 257

Laskar, J. 1993b, Cel. Mech. and Dyn. Astr., 56, 191

Laskar, J., \& Robutel, P. 1995, Cel. Mech. and Dyn. Astr., 62, 193

Laskar, J., Froeschlè, C., \& Celletti, A. 1992, Physica D, 56, 253

Laughlin, G., \& Chambers, J. E. 2001, ApJ, 55, L109

Lee, M. H., \& Peale, S. J. 2002, ApJ, 567, 596

Lin, D. N. C., \& Papaloizou, J. C. B. 1986, ApJ, 309, 846

Marzari, F., Scholl, H., \& Tricarico, P. 2005, A\&A, 442, 359

Michtchenko, T. A., \& Malhotra, R. 2004, Icarus, 168, 237

Moorhead, A. V., \& Adams, F. C. 2005, Icarus, 178, 517

Murray, C. D., \& Dermott S. F. 1999, Solar System Dynamics (New York: Cambridge University Press)

Murray, N., Hansen, B., Holman, M., \& Tremaine, S. 1998, Science, 279, 69

Nelson, R. P., \& Papaloizou, J. C. B. 2002, MNRAS, 333, L26

Rasio, F. A., \& Ford, E. B. 1996, Science, 274, 954

Sidlichovský, M., \& Nesvorný, D. 1997, Cel. Mech. and Dyn. Astr., 65, 137

Tanaka, H., Takeuchi, T., \& Ward, W. R. 2002, ApJ, 565, 1257

Tsiganis, K., Gomes, R., Morbidelli, A., \& Levison, H. F. 2005, Nature, 435, 459

Ward, W. R. 1997, Icarus, 126, 261

Weidenschilling, S. J., \& Marzari, F. 1996, Nature, 384, 619 